\title{
Detecção e influência de Fusarium spp. na qualidade fisiológica de sementes de pepino
}

\author{
Detection and influence of Fusarium spp. in physiological quality of cucumber seeds
}

\section{Vanessa Ocom Menezes ${ }^{*}$ Daniele Cardoso Pedroso $^{\mathrm{I}}$ Graziela Piveta $^{\mathrm{I}}$ Marlove Fátima Brião Muniz $^{\mathrm{I}}$ Nilson Lemos de Menezes ${ }^{\mathrm{II}}$ Danton Camacho Garcia ${ }^{\mathrm{II}}$ Luciana Zago Ethur ${ }^{\mathrm{III}}$ Ricardo Feliciano dos Santos ${ }^{\mathrm{I}}$ Lilian Madruga Tunes ${ }^{\mathrm{I}}$}

\section{RESUMO}

Este trabalho teve por objetivo isolar, identificar e selecionar isolado(s) de Fusarium spp. em sementes de pepino e verificar a sua influência na qualidade fisiológica. Foram utilizados três sublotes e os tratamentos consistiram em: testemunha absoluta; $B D A+$ manitol - 0,8Mpa; $B D A ; B D A+$ manitol - 0,8Mpa + fungo; e BDA + fungo. A qualidade fisiológica foi avaliada através de testes em laboratório e casa de vegetação. Cinco isolados de Fusarium verticillioides foram identificados e a sua transmissão através das sementes para plantas não foi possível afirmar. A técnica da restrição hídrica foi eficiente para proporcionar infecção das sementes, sabendo que e o fungo influencia negativamente na qualidade fisiológica das sementes de pepino da cultivar Caipira.

Palavras-chave: Cucumis sativus L., Fusarium verticillioides, inoculação, estresse hídrico.

\section{ABSTRACT}

This research had the objective of isolate, identifying and select isolates (s) of Fusarium spp. in cucumber seeds and checking the influence of this (these) isolate(s) on the physiological quality. Three batches were used and treatments utilized consisted in: absolute witness; PDA + mannitol $0.8 M$ pa; PDA pure; PDA + mannitol - 0.8Mpa + fungi; and $P D A+$ fungi. The physiological quality was assessed by tests carried out in laboratory and greenhouse. Five isolates off Fusarium verticillioides were isolated and it was not possible to affirm its transmission through seed. The hydric restriction technique was efficient to provide the infection of cucumber seeds, since this fungus affected the physiological quality of cucumber seeds of this cultivar.
Key words: Cucumis sativus L., Fusarium verticillioides, inoculation, hydric stress.

\section{INTRODUÇÃO}

O bom desempenho de uma cultura depende da qualidade das suas sementes, pois elas contêm todo o potencial genético que a planta pode expressar. No entanto, esse desempenho pode variar entre lotes de uma mesma espécie ou cultivar, dependendo dos seus aspectos físicos, sanitários, genéticos e fisiológicos (GUIMARÃES et al., 2006).

Entre vários fatores que podem influenciar a qualidade das sementes, está a associação com microrganismos. Espécies de Fusarium são agentes causais da mancha do fusário no pepineiro (Cucumis sativus L.), atacando a planta em qualquer estádio de desenvolvimento, o que ocasiona tombamento e infecção de plantas velhas, resultando na murcha de brotos e de toda a planta, além da descoloração vascular de raiz e caule (ZITTER et al., 2006).

Para auxiliar nos estudos sobre Fusarium spp. associados às sementes, há necessidade de colocá-las com o fungo, fazendo-se necessária a inoculação artificial, de modo a garantir a expressão e sintomatologia da doença, pois há dificuldade na

'Departamento de Defesa Fitossanitária, Universidade Federal de Santa Maria (UFSM), Av. Roraima, 1000, 97105-900, Santa

Maria, RS, Brasil. E-mail: vane_menezes@yahoo.com.br. *Autor para correspondência.

IIDepartamento de Fitotecnia, UFSM, Santa Maria, RS, Brasil.

"Inniversidade Federal do Pampa (UNIPAMPA), Itaqui, RS, Brasil. 
obtenção de sementes com diferentes níveis de infecção natural. Atualmente, tem sido utilizada a técnica da restrição hídrica, que inibe a germinação sem afetar o desenvolvimento dos fungos, utilizando baixos potenciais hídricos, através da adição de solutos ao substrato em que haverá inoculação. Há outras técnicas, como: imersão de sementes em suspensão de conídios e/ou hifas; apenas seu revestimento externo com esporos; e o contato das sementes com a colônia fúngica em meio de cultura. Por esses métodos, entretanto, os fungos ficam aderidos ao tegumento das sementes e a infecção não é assegurada. Outro fator limitante seria o tempo de exposição das sementes à colônia, pois elas podem iniciar a germinação, conforme a espécie avaliada (MACHADO et al., 2001a).

Nessa perspectiva, o presente trabalho objetivou isolar, identificar e selecionar isolado (s) de Fusarium spp.. do pepineiro, cultivar 'Caipira', e verificar a influência desse (s) isolado (s) na qualidade fisiológica e fitossanitária das sementes, através da técnica de inoculação com restrição hídrica.

\section{MATERIAL E MÉTODOS}

Um lote de sementes de pepino, cultivar 'Caipira', foi dividido em três sublotes para obtenção de diferentes níveis de qualidade fisiológica, através do envelhecimento artificial, conduzido em caixas plásticas tipo gerbox (AOSA, 1983), por períodos de zero, 12 e 36h (sublotes 1, 2 e 3, respectivamente). Após, as sementes foram submetidas aos testes de germinação, sanidade (Blotter Test) e determinação do teor de umidade para avaliação de sua qualidade inicial.

A partir do Blotter Test, foram utilizados cinco isolados de Fusarium spp., obtidos das sementes dos três sublotes, que foram colocados em placas de Petri contendo meio de cultura BDA e repicados até a obtenção de colônias puras. Foi feita a identificação dos isolados conforme a técnica de cultura monospórica, descrita por FERNANDES (1993), seguindo a chave de classificação de VENTURA (2000). Todas as sementes utilizadas no teste sanitário foram analisadas e se constatou a presença de uma espécie infectando-as: Fusarium verticillioides (Sacc.) Nirenberg.

As inoculações $\operatorname{com} \boldsymbol{F}$. verticillioides foram realizadas utilizando-se como substrato o meio de cultura BDA, modificado pela adição de $33,10 \mathrm{~g} \mathrm{~L}^{-1} \mathrm{de}$ manitol, segundo COUTINHO et al. (2001). O cálculo para obtenção da quantidade de soluto necessário para o potencial hídrico de $-0,8 \mathrm{Mpa}$ foi obtido através da fórmula de Van't Hoff, citada por SOUZA \& CARDOSO (2000). Em seguida, o fungo foi repicado para placas de
Petri com o meio modificado, conforme a metodologia descrita por MACHADO et al. (2001a), permanecendo na placa por um período de $48 \mathrm{~h}$.

Foram aplicados cinco tratamentos em cada sublote: T1: testemunha absoluta sem restrição hídrica (0Mpa), as sementes não foram dispostas previamente em meio de cultura; T2: sementes dispostas em meio de cultura BDA + manitol; T3: sementes dispostas sobre o meio de cultura BDA, sem manitol, o qual apresenta um potencial hídrico-0,35Mpa (WEARING \& BURGUESS, 1979); T4: sementes dispostas sobre meio de cultura BDA + manitol $+\boldsymbol{F}$. verticillioides; $\mathrm{T} 5$ : sementes dispostas sobre meio de cultura BDA $+\boldsymbol{F}$. verticillioides, sem restrição hídrica. Após os tratamentos, as sementes foram submetidas às avaliações em laboratório e casa de vegetação.

Foram conduzidos quatro testes em laboratório:

1) germinação -conduzido com quatro repetições de 50 sementes, semeadas em rolos de papel umedecidos com água destilada no volume equivalente a 2,5 vezes a massa do papel seco e, posteriormente, colocadas em germinador à temperatura de $25^{\circ} \mathrm{C}$ e fotoperíodo de $12 \mathrm{~h}$. As avaliações foram realizadas aos quatro e oito dias após a semeadura, sendo contabilizadas as porcentagens de plântulas normais, anormais e sementes mortas (BRASIL, 1992);

2) teste de frio - a semeadura foi feita conforme a metodologia do teste de germinação, porém, os rolos foram acondicionados em sacos plásticos e permaneceram cinco dias em câmara BOD, à temperatura de $10^{\circ} \mathrm{C}$. Após, foram colocados em germinador a $25^{\circ} \mathrm{C}$, durante cinco dias, considerandose a porcentagem de plântulas normais;

3) crescimento de plântulas - foram utilizadas quatro repetições de 20 sementes, sendo a semeadura no terço superior do papel umedecido com água destilada, no volume de 2,5 vezes a massa do papel seco. Os rolos foram mantidos no germinador por cinco dias, à temperatura de $25^{\circ} \mathrm{C}$. Após, foi feita a separação de 10 plântulas em quatro repetições, sendo submetidas às medições com régua milimetrada, com resultados expressos em centímetros (BRASIL, 1992). Para obtenção dos dados de comprimento de hipocótilo, foram realizadas as medidas da zona de diferenciação entre radícula/hipocótilo até os cotilédones e, para a radícula foi considerado o comprimento da raiz primária.

4) qualidade sanitária: a avaliação sanitária foi realizada através do Blotter Test, com oito repetições de 25 sementes, distribuídas em caixas gerbox, utilizando-se duas folhas de papel filtro umedecidas com água estéril na proporção de 2,5 vezes a massa do papel seco. As caixas foram mantidas em câmara de 
crescimento a $25^{\circ} \mathrm{C}$ em regime alternado de $12 \mathrm{~h}$ de luz, por sete dias. Em seguida, as sementes foram examinadas individualmente, com auxílio de microscópio estereoscópico e óptico, para verificação da presença de fungos, que foram identificados ao nível de gênero (BARNETT \& HUNTER, 1998). Os resultados foram expressos em porcentagem de sementes contaminadas.

Em casa de vegetação, foram conduzidos três testes:

1) emergência - foram utilizadas bandejas plásticas contendo substrato Plantmax ${ }^{\circledR}$, realizado com quatro repetições de 25 sementes. As irrigações foram diárias e a avaliação foi feita aos 21 dias após a semeadura, computando-se a porcentagem de plântulas normais emergidas. As sementes mortas ou que não germinaram foram transferidas para caixas gerbox desinfestadas previamente com $\mathrm{NaClO}$ e álcool etílico $70 \%$, utilizando-se a metodologia do Blotter Test para verificar a presença de estruturas de F. verticillioides;

2) crescimento de plantas - realizado juntamente com o teste de emergência, foram utilizadas quatro repetições de 10 plantas, retiradas de forma aleatória aos 21 dias após a semeadura. Para obtenção dos dados de comprimento de hipocótilo e raiz, foi utilizada a metodologia para o teste já descrito em laboratório;

3) índice de velocidade de emergência (IVE) - determinado utilizando-se quatro repetições de 25 sementes, efetuando-se contagens diárias de plântulas emersas até obter-se número constante. Para cada repetição, foi calculado o IVE, conforme MAGUIRE (1962).

O delineamento experimental utilizado foi inteiramente casualizado (DIC) com quatro repetições, constituindo um bifatorial $5 \times 3$, correspondendo aos tratamentos e aos sublotes de sementes de pepino. A análise estatística dos resultados foi realizada utilizando-se o Software Sistema de Análises Estatísticas-SANEST (ZONTA et al., 1986). As médias foram comparadas pelo Teste de Tukey em nível de $5 \%$ de probabilidade de erro.

\section{RESULTADOS E DISCUSSÃO}

Os sublotes apresentaram diferentes porcentagens quanto aos graus de umidade das sementes. Aquele que não foi submetido ao envelhecimento acelerado apresentou um percentual de umidade de $6,71 \%$. Já, as sementes dos sublotes 2 e 3 apresentaram grau de umidade de 18,83 e 29,83\%, respectivamente. Segundo DESAI et al. (1997), graus de umidade superiores a $20 \%$ podem promover o aquecimento da massa de sementes a uma temperatura letal. Além disso, o aumento do grau de umidade leva a um aumento na taxa respiratória e propicia a ação de microrganismos. A porcentagem de germinação dos sublotes 1, 2 e 3 foi 98,54, 97,38 e 86,58\%, respectivamente, demonstrando a alta qualidade fisiológica de cada um. Esses dados não foram analisados estatisticamente e serviram apenas como base para caracterização inicial da qualidade dos sublotes.

$\mathrm{Na}$ análise sanitária, foram detectados os seguintes fungos: Fusarium verticillioides, Penicillium spp., Alternaria spp. e Aspergillus spp.. Como o objetivo do trabalho é o patógeno F.verticillioides, a discussão será baseada apenas nesse fungo. $\boldsymbol{F}$. verticillioides foi detectado nos três sublotes, com maior porcentagem naquele de qualidade intermediária. MENEZES et al. (2008), trabalhando com diferentes tempos de envelhecimento acelerado em sementes de zínia, encontraram maior incidência desse fungo em sementes mais úmidas.

Os resultados referentes aos testes de germinação e teste de frio estão na tabela 1. Verificouse que houve interação entre os fatores para todas as variáveis, com exceção do teste de frio, indicando a dependência do efeito dos tratamentos aplicados às sementes, da qualidade fisiológica estabelecida pelos sublotes.

As médias foram relativamente altas em todos os tratamentos no teste de germinação, havendo uma diferenciação estatística naqueles tratamentos com inoculação nos três sublotes avaliados, demonstrando que, para a cultivar, a inoculação reduziu a germinação. Esses dados corroboram os encontrados para esse mesmo teste, também em sementes de pepino, porém para a cultivar 'Wisconsin' (MENEZES, 2009).

Em relação a plântulas anormais, as maiores porcentagens ocorreram nos tratamentos suplementados com manitol e naqueles com fungo. É importante observar que no tratamento testemunha não houve plântulas anormais em dois sublotes, demonstrando a alta qualidade fisiológica desses. Para todos os tratamentos, o sublote 2 manteve a mesma tendência do primeiro sublote. Para o sublote de maior grau de umidade, apenas o tratamento 5 diferiu dos demais, o que aponta a capacidade de $\boldsymbol{F}$. verticillioides em causar danos ao pepineiro, afetando a capacidade de formação de plântulas normais em condições de maior umidade.

A incidência de sementes mortas apresentou maior relevância no sublote 1 , e as maiores porcentagens ocorreram nos tratamentos com BDA e com BDA + fungo. $\mathrm{O}$ tratamento com a presença de $\mathrm{F}$. 
Tabela 1 - Qualidade fisiológica dos sublotes de sementes de pepino, cultivar Caipira, submetidas a diferentes tratamentos. Santa Maria - RS, 2008.

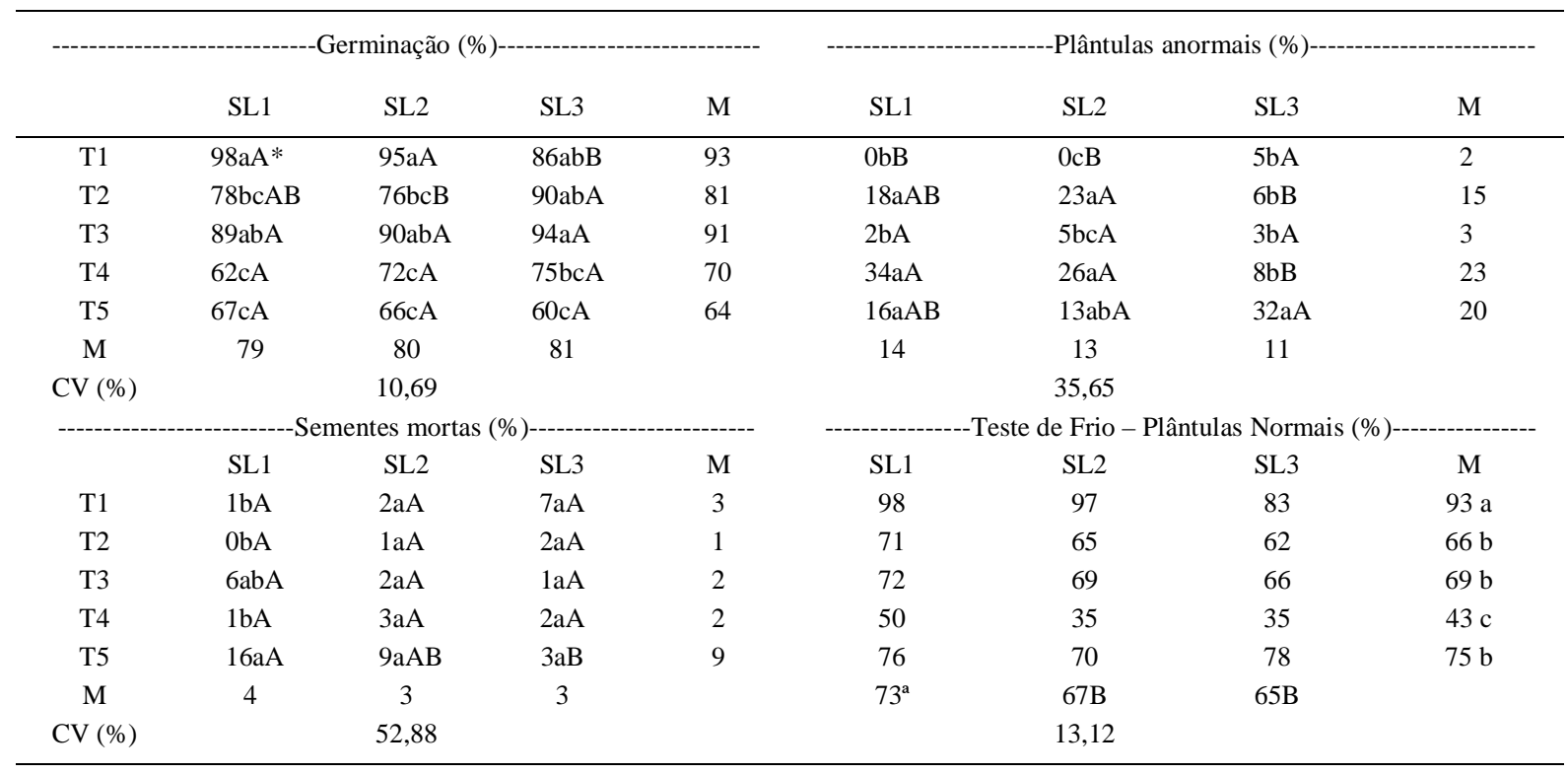

* Médias seguidas pela mesma letra maiúscula na linha e minúscula na coluna não diferem entre si pelo teste de Tukey, em nível de 5\% de probabilidade de erro. SL1: sublote 1 - envelhecimento acelerado por zero h; SL2: sublote 2 - envelhecimento acelerado por 24h; SL3: sublote 3 - envelhecimento acelerado por 48h; M: média; T1: Testemunha; T2: BDA + manitol; T3: BDA; T4: BDA + manitol + F verticillioides; T5: BDA + F. verticillioides.

verticillioides corrobora o que MACHADO et al. (2004) concluíram em trabalhos com sementes de algodão: que o uso da inoculação de fungos através da restrição hídrica aumenta o número de sementes mortas, devido ao alto índice de infecção alcançado pela técnica. Nesse trabalho, nos demais sublotes não ocorreu diferença estatística em relação aos tratamentos utilizados, mas houve a presença de sementes mortas em todos.

No teste de frio, a testemunha diferiu dos demais, com uma alta média de plântulas normais. $O$ tratamento utilizando BDA + fungo obteve a menor porcentagem de plântulas normais, diferindo dos demais. A baixa disponibilidade de água no meio, além da inoculação de $\boldsymbol{F}$. verticillioides, certamente contribuiu para esse resultado. $\mathrm{O}$ fato de o tratamento com manitol e fungo não ter diferido daqueles sem fungo pode ser explicado fitopatologicamente, pois as sementes podem proteger o fungo dos estresses do ambiente, sabendo que a exposição da semente ao teste de frio caracteriza uma adversidade. Para esse teste, foram analisadas, estatisticamente, as médias e não os valores obtidos para cada tratamento sobre cada sublote de sementes, pois não houve interação estatística entre esses fatores.

Resultados referentes aos testes de comprimento de plântulas encontram-se na tabela 2.
Para a variável comprimento de hipocótilo, os três sublotes obtiveram, praticamente, os mesmos resultados para os tratamentos utilizados, sendo a testemunha a única a diferir, diminuindo seus valores conforme o aumento da umidade das sementes, ou seja, conforme diminuiu a qualidade do sublote. Apenas no sublote 2, o tratamento com BDA não diferiu da Testemunha. Esses resultados demonstram que, para a cultivar, até mesmo o tratamento apenas com BDA afeta o comprimento de hipocótilo das plântulas, ressaltando que, no sublote 3 , esse tratamento teve uma média muito baixa. ÀVILA et al. (2007), pesquisando a influência do potencial hídrico em sementes de canola, constatou que, no potencial osmótico de - 0,50MPa, a redução do comprimento do hipocótilo foi de aproximadamente $50 \%$.

Para a variável comprimento de raiz, os tratamentos com fungo obtiveram médias inferiores às dos outros tratamentos nos três sublotes, ressaltando que, no sublote de menor média, ratificou os resultados obtidos para a variável anterior. Sabe-se que Fusarium spp. é um patógeno vascular que pode causar atrofiamento do sistema vascular de plântulas.

Na tabela 3, encontram-se os resultados dos testes de emergência e IVE. No teste de emergência, não houve interação entre os fatores avaliados, 
Tabela 2 - Crescimento de plântulas utilizadas para avaliação da qualidade fisiológica em sublotes de pepino, cultivar Caipira, submetidas a diferentes tratamentos. Santa Maria - RS, 2008.

\begin{tabular}{|c|c|c|c|c|c|c|c|c|}
\hline & SL1 & SL2 & SL3 & M & SL1 & SL2 & SL3 & M \\
\hline $\mathrm{T} 1$ & $7,54 \mathrm{aA} *$ & $4,84 \mathrm{aB}$ & $4,87 \mathrm{aB}$ & 5,75 & $12,59 \mathrm{aA}$ & $9,16 \mathrm{aB}$ & $7,48 \mathrm{aB}$ & 9,74 \\
\hline $\mathrm{T} 2$ & $3,09 \mathrm{bA}$ & $2,52 \mathrm{bA}$ & $2,31 \mathrm{bA}$ & 2,64 & $5,75 \mathrm{bA}$ & $7,25 \mathrm{abA}$ & $6,83 \mathrm{abA}$ & 6,61 \\
\hline $\mathrm{T} 3$ & $3,35 \mathrm{bB}$ & $4,99 \mathrm{aA}$ & $1,03 \mathrm{bC}$ & 3,12 & $5,77 \mathrm{bB}$ & $9,03 \mathrm{aA}$ & $1,19 \mathrm{dC}$ & 5,33 \\
\hline $\mathrm{T} 4$ & $2,74 \mathrm{bA}$ & $2,87 \mathrm{bA}$ & $2,31 \mathrm{bA}$ & 2,64 & $2,80 \mathrm{cB}$ & $5,68 \mathrm{bA}$ & $4,60 \mathrm{bcAB}$ & 4,36 \\
\hline $\mathrm{T} 5$ & $3,35 \mathrm{bA}$ & $2,87 \mathrm{bAB}$ & $1,79 \mathrm{bB}$ & 2,67 & $5,77 \mathrm{bA}$ & $5,54 \mathrm{bA}$ & $2,79 \mathrm{~dB}$ & 4,70 \\
\hline M & 4,01 & 3,62 & 2,46 & & 6,54 & 7,33 & 4,58 & \\
\hline $\mathrm{CV}(\%)$ & & 25,03 & & & & 20,97 & & \\
\hline
\end{tabular}

*Médias seguidas pela mesma letra maiúscula na linha e minúscula na coluna não diferem entre si pelo teste de Tukey, em nível de 5\% de probabilidade de erro. SL1: sublote 1 - envelhecimento acelerado por zero h; SL2: sublote 2 - envelhecimento acelerado por 24h; SL3: sublote 3 - envelhecimento acelerado por 48h; M: média; T1: Testemunha; T2: BDA + manitol; T3: BDA; T4: BDA + manitol + $\boldsymbol{F}$. verticillioides; T5: BDA + F. verticillioides.

mostrando a independência do efeito dos tratamentos em relação à qualidade fisiológica dos sublotes utilizados, fato que também ocorreu em condições de laboratório para o teste de frio. Verifica-se também, uma queda geral nas médias de germinação em campo, quando comparadas ao laboratório.

$\mathrm{O}$ teste de emergência e IVE mostraram resultados semelhantes. As maiores médias foram obtidas nas sementes do tratamento testemunha e as menores em todos os sublotes, no tratamento 4 , demonstrando o efeito da restrição hídrica nesses testes, em condições semi-controladas para esse patossistema. Outro estudos revelaram que sementes de soja, inoculadas através da restrição hídrica com Sclerotinia sclerotiorum, num potencial de $-1,0 \mathrm{MPa}$, tiveram resultado letal para todas as sementes (MACHADO, et al., 2001b). É Interessante observar que, no teste de IVE, o tratamento com BDA + fungo,

Tabela 3 - Porcentagem de emergência e crescimento de plantas de sublotes de sementes de pepino, cultivar 'Caipira', submetidas a diferentes tratamentos. Santa Maria - RS, 2008.

\begin{tabular}{|c|c|c|c|c|c|c|c|c|}
\hline \multicolumn{5}{|c|}{-------------------------Emergência (\%)-------------------------- } & \multicolumn{4}{|c|}{-Índice de velocidade de emergência-- } \\
\hline & SL1 & SL2 & SL3 & M & SL1 & SL2 & SL3 & M \\
\hline T1 & $59^{*}$ & 66 & 51 & $58 \mathrm{a}$ & $10,71 \mathrm{aB}$ & $15,53 \mathrm{aA}$ & $10,56 \mathrm{aB}$ & 12,20 \\
\hline $\mathrm{T} 2$ & 50 & 43 & 31 & $41 \mathrm{~b}$ & $8,98 \mathrm{abA}$ & $6,88 \mathrm{bA}$ & 7,63abA & 7,83 \\
\hline $\mathrm{T} 3$ & 63 & 61 & 45 & $56 \mathrm{a}$ & $12,01 \mathrm{aA}$ & $9,56 \mathrm{bAB}$ & $7,39 \mathrm{aB}$ & 9,65 \\
\hline $\mathrm{T} 4$ & 28 & 18 & 13 & $19 \mathrm{c}$ & $3,60 \mathrm{cA}$ & $3,36 \mathrm{cA}$ & $1,64 \mathrm{cA}$ & 2,89 \\
\hline T5 & 45 & 32 & 30 & $36 \mathrm{~b}$ & $7,62 \mathrm{bA}$ & $7,07 \mathrm{bA}$ & $5,21 \mathrm{bA}$ & 6,63 \\
\hline M & $49 \mathrm{~A}$ & $44 \mathrm{~A}$ & $33 \mathrm{~A}$ & & 8,60 & 8,44 & 6,49 & \\
\hline $\mathrm{CV}(\%)$ & & 9,69 & & & \multicolumn{4}{|c|}{19,26} \\
\hline \multicolumn{5}{|c|}{ - Comprimento de hipocótilo $(\mathrm{cm})$} & ------- & Comprime & raiz $(\mathrm{cm})$ & ---- \\
\hline & SL1 & SL2 & SL3 & M & SL1 & SL2 & SL3 & M \\
\hline $\mathrm{T} 1$ & $4,57 \mathrm{aA}$ & $3,97 \mathrm{aB}$ & $3,25 \mathrm{aB}$ & 2,93 & $8,89 \mathrm{aA}$ & $7,31 \mathrm{bA}$ & $5,85 \mathrm{aC}$ & 7,35 \\
\hline $\mathrm{T} 2$ & $3,10 \mathrm{bA}$ & $2,98 \mathrm{aA}$ & $2,92 \mathrm{aA}$ & 3,00 & $5,44 \mathrm{bA}$ & $5,13 \mathrm{aB}$ & $4,72 \mathrm{bA}$ & 5,10 \\
\hline $\mathrm{T} 3$ & $3,25 \mathrm{bA}$ & $2,18 \mathrm{cB}$ & $3,10 \mathrm{aA}$ & 2,84 & $5,85 \mathrm{bA}$ & $5,19 \mathrm{bA}$ & $5,44 \mathrm{abA}$ & 5,49 \\
\hline $\mathrm{T} 4$ & $2,25 \mathrm{cAB}$ & $2,15 \mathrm{cB}$ & $2,79 \mathrm{aA}$ & 2,40 & $3,28 \mathrm{cB}$ & $3,18 \mathrm{bC}$ & $5,77 \mathrm{aA}$ & 4,08 \\
\hline $\mathrm{T} 5$ & $2,30 \mathrm{cA}$ & $2,35 \mathrm{bcA}$ & $2,67 \mathrm{aA}$ & 2,44 & $3,37 \mathrm{cB}$ & $2,99 \mathrm{bC}$ & $5,25 \mathrm{aBA}$ & 3,87 \\
\hline M & 3,09 & 2,73 & 2,95 & & 5,37 & 4,76 & 5,41 & \\
\hline $\mathrm{CV}(\%)$ & & 11,72 & & & & 8,15 & & \\
\hline
\end{tabular}

*Médias seguidas pela mesma letra maiúscula na linha e minúscula na coluna não diferem entre si pelo teste de Tukey, em nível de 5\% de probabilidade de erro. SL1: sublote 1 - envelhecimento acelerado por zero h; SL2: sublote 2 - envelhecimento acelerado por 24h; SL3: sublote 3 - envelhecimento acelerado por 48h; M: média; T1: Testemunha; T2: BDA + manitol; T3: BDA; T4: BDA + manitol + $\boldsymbol{F}$. verticillioides; $\mathrm{T} 5 \mathrm{BDA}+\boldsymbol{F}$. verticillioides. 
principalmente nos sublotes de melhores qualidades fisiológicas, seus valores diferiram da Testemunha, mas, mesmo assim, foram bem significativos em relação aos demais. Sabe-se que, mesmo em sementes de mesmo lote, há discrepâncias de qualidade, o que pode ter acontecido no presente trabalho. O IVE baseia-se no princípio de que a velocidade de emergência das plântulas em campo é proporcional ao vigor das sementes (MARCOS FILHO et al., 1987).

Os resultados referentes ao comprimento de hipocótilo demonstram que, para os sublotes 1 e 2 , as menores médias apareceram nos tratamentos com fungo, embora o tratamento com BDA puro, no sublote 2 , não diferiu dos inoculados. SANTOS et al. (2004) verificaram, utilizando dois sublotes de feijão, que o comprimento de hipocótilo diminuiu em função da qualidade das sementes.

Para a variável comprimento de raiz, nos sublotes 1 e 2, as menores médias também apareceram nos tratamentos com o fungos, porém, no sublote 2 , diferiram apenas da Testemunha, o que demonstra que os outros tratamentos também influenciaram no comprimento de raiz, porém em menor intensidade em relação à inoculação. No sublote 3 , os tratamentos com fungo não diferiram da Testemunha, sendo que apenas o tratamento com BDA diferiu dos demais e apresentou a menor média. Esse fato contradiz o exposto por ÀVILA et al. (2007), os quais afirmam que o aumento no potencial osmótico ocasiona um decréscimo significativo no comprimento das plântulas de milho.

\section{CONCLUSÃO}

Pode-se inferir que Fusarium verticillioides influencia negativamente na qualidade fisiológica das sementes de pepino da cultivar 'Caipira'.

\section{REFERÊNCIAS}

ASSOCIATION OF OFFICIAL SEED ANALYSTS - AOSA. Seed vigor testing handbook. East Lansing, 1983. 93p.

ÁVILA, M.R. et al. Influência do estresse hídrico simulado com manitol na germinação de sementes e crescimento de plântulas de canola. Revista Brasileira de Sementes, v.29, n.1, p.98-106, 2007. Disponível em: <http://www.scielo.br/ pdf/rbs/v29n1/14.pdf >. Acesso em: 23 dez. 2008. doi: 10.1590/ S0101-31222007000100014.

BARNETT, H.L.; HUNTER, B.B. Illustrated genera of imperfect fungi. St Paul, Minnesota: PS, 1998. 218p.

BRASIL. Ministério da Agricultura e Reforma Agrária. Regras para análise de sementes. Brasília: SNDA/DNDV/CLAV, 1992. 365p.
COUTINHO, M.C. et al. Uso da restrição hídrica na inibição ou retardamento da germinação de sementes de arroz e feijão submetidas ao teste de sanidade em meio agar-água. Revista Brasileira de Sementes, v.23, n.2, p.127-135, 2001. Disponível em: <http://www.abrates.org.br/revista/artigos/2001/ v23n2/artigo19.pdf>. Acesso em: 19 nov. 2007.

DESAI, B.B. et al. Seeds handbook biology, production, processing and storage. New York: Basel, 1997. 627p.

FERNANDES, M.R. Manual para laboratório de fitopatologia. Passo Fundo: EMBRAPA-CNPT, 1993. 128p.

GUIMARÃES, R.M. et al. Aspectos fisiológicos de sementes. Informe Agropecuário, v.27, n.232, p.40-50, 2006.

MACHADO, J.C. et al. Uso da restrição hídrica na inoculação de fungos em sementes de milho. Revista Brasileira de Sementes, v.23, n.2, p.88-94, 2001a. Disponível em: <http:/ /www.abrates.org.br/revista/artigos/2001/v23n2/artigo12.pdf>. Acesso em: 23 dez. 2008.

MACHADO, J.C. et al. Inoculação artificial de sementes de soja por fungos utilizando solução de manitol. Revista Brasileira de Sementes, v.23, n.2, p.95-101, 2001b. Disponível em: <http://www.abrates.org.br/revista/artigos/2001/ v23n2/artigo13.pdf. Acesso em: 15 jan. 2009.

MACHADO, J.C. et al. Uso da restrição hídrica na inoculação de fungos em sementes de algodoeiro (Gossypium hirsutum L.). Revista Brasileira de sementes, v.26, n.3, p.62-67, 2004. Disponível em: <http://www.scielo.br/pdf/rbs/v26n1/ a10v26n1.pdf. Acesso em: 15 jan. 2009.

MAGUIRE, J.D. Spead of germination-aid in selection and evaluation for seedling emergence and vigour. Crop Science, v.2, n.1, p.176-177, 1962.

MARCOS FILHO, J. et al. Avaliação da qualidade das sementes. Piracicaba: FEALQ, 1987. 230p.

MENEZES, V.O. Inoculação de Fusarium moniliforme (Sheld.) em sementes de duas cultivares de pepino através da técnica da restrição hídrica e sua influência sobre a qualidade fisiológica. 2009. 85f. Dissertação (Mestrado em Agronomia) - Curso de Pós-graduação em Agronomia, Universidade Federal de Santa Maria, RS.

MENEZES, V.O. et al. Envelhecimento acelerado em sementes de Zinnia elegans Jacq. colhidas em diferentes épocas. Revista Brasileira de Sementes, v.30, n. 3, p.39-47, 2008. Disponível em: 〈http://www.scielo.br/pdf/rbs/v30n3/06.pdf>. Acesso em: 17 jul. 2009. doi: 10.1590/S010131222008000300006 .

SANTOS, C.M.R. et al. Alterações fisiológicas e bioquímicas em sementes de feijão envelhecidas artificialmente. Revista Brasileira de Sementes, v.26, n.1, p.110-119, 2004. Disponível em: <http://www.scielo.br/pdf/rbs/v26n1/ a17v26n1.pdf . Acesso em: 15 out. 2009. doi: 10.1590/S010131222004000100017 .

SOUZA, G.M.; CARDOSO, V.J.M. Effects of different environmental stress on seed germination. Seed Science Technology, v.28, n.3, p.621-630, 2000. 
WEARING, A.H.; BURGESS, L.W. Water potential and the saprophytic growth of Fusarium roseum "graminearum". Soil Biology and Biochemistry, v.11, n.6, p.661-667, 1979.

VENTURA, J.A. Taxonomia de Fusarium e seus segredos. Parte II - chaves para identificação. Revisão Anual de Patologia de Plantas, v.8, p.303-338, 2000.
ZITTER, T.A. et al. Compendium of cucurbit diseases. Saint Paul, Minnesota: American Phytopathological Society - APS, 1996. $87 \mathrm{p}$.

ZONTA, E.P.; MACHADO, A.A. Sistema de análise estatística para microcomputadores - SANEST. Pelotas: UFPel, 1986. 150p. 